of the Japan Society for Applied Physics on diamond synthesis and applications.

Some of the first diamond film products from the Japanese company Sumitomo Electric include an acoustic speaker element of titanium or aluminium which is coated with about $1 \mu \mathrm{m}$ of diamond to improve the high-frequency response. Another is the first step towards a possible diamond semiconductor, consisting of p-type $8 \mathrm{~mm} \times 8 \mathrm{~mm} \times 4 \mathrm{~mm}$ doped yellow synthetic (high-pressure) diamonds, which can be used for substrates for the epitaxial overgrowth of an n-type layer. But perfect single-crystal p-type films and active semiconductor elements may take several years to develop.

Rustum Roy is former Evan Pugh Professor of the Solid State and Director of the Materials Research Laboratory, Pennsylvania State University, Pennsylvania 16802, USA.

\title{
Metabolism
}

\section{New approaches to vitamin D}

\section{Ian Dickson}

A DECADE or so ago our understanding of the main features of the vitamin D system seemed to be almost complete. But it is clear that the subject is far more complicated than was originally thought, and what seemed earlier a plausible interpretation of its role and mechanisms of action looks increasingly incompatible from the new evidence. It is proving frustratingly difficult to produce a revised hypothesis that will accomodate all these data or even to understand the reasons why symptoms of vitamin $D$ deficiency develop in many clinical conditions. If the observations by Clements and co-workers reported on page 62 of this issue ${ }^{1}$, which are strongly supported by a recent report from another laboratory ${ }^{2}$, can be extrapolated from rats to humans, as their preliminary studies and also observations by others ${ }^{3}$ suggest, an empirical explanation for some of these disorders could be within reach.

The first significant step in understanding the mechanism of vitamin $\mathrm{D}$ action was the finding ${ }^{4}$ in 1968 that it is converted by a hydroxylation in the liver to the more active form 25 -hydroxyvitamin $\mathrm{D}_{3}$. It was soon discovered that 25 -hydroxyvitamin $\mathrm{D}_{3}$ is converted by a second hydroxylase, located in the kidney and regulated by parathyroid hormone, to 1,25 dihydroxyvitamin $D_{3}$, an even more active form which stimulates calcium absorption and synthesis of a calcium-binding protein by the intestine. This metabolite was the most potent bone-resorbing agent then known. These data were combined to produce a general explanation ${ }^{5}$ of the action and physiological function of vitamin $\mathrm{D}$, in which the calcium-regulating hormone 1,25-dihydroxyvitamin $\mathrm{D}_{3}$ was thought to act through the cell nucleus on two main target tissues, bone and intestine, in the manner of a steroid hormone. A fall in blood calcium would thus trigger the secretion of parathyroid hormone from the parathyroid gland which would stimulate the 1-hydroxylase in the kidney to produce more 1,25 -dihydroxyvitamin $\mathrm{D}_{3}$. The latter hormone would restore normal plasma calcium levels by stimulating calcium absorption by the gut or by bone resorption. The classic symptom of vitamin D deficiency, the disorder of bone formation as seen in rickets or its adult form osteomalacia, was widely believed to be an indirect effect of the abnormally low plasma levels of calcium and phosphate.

We now know that the situation is more complex. Cellular binding proteins or receptors for 1,25-dihydroxyvitamin $\mathrm{D}_{3}$ are not confined to the classical target tissues such as intestine and bone but are present in many tissues ${ }^{6}$ including brain, cartilage, kidney, mammary gland, muscle, ovary, placenta, parathyroid, parotid, pancreas, pituitary, skin, teeth, testes, thymus and uterus as well as macrophages, monocytes and activated lymphocytes. It may therefore be more appropriate to think of 1,25 dihydroxyvitamin $\mathrm{D}_{3}$ as fundamental to many different types of cell, where it probably has a role in regulating intracellular calcium, than specialized to a few. It may be somewhat restrictive to think of it as a hormone that regulates calcium homoeostasis as this property may be only incidental to its primary role.

Recent evidence suggests that 1,25 dihydroxyvitamin $D_{3}$ does not act only through the cell nucleus to alter gene transcription in the manner of a steroid hormone but also influences cellular metabolism by alternative, more rapid mechanisms. There are changes in the levels of certain enzymes involved in polyamine biosynthesis in duodenal cells as early as $\mathbf{3 0}$ minutes after administering the metabolite to rachitic chickens ${ }^{7}$, and calcium transport in perfused duodena from normal chicks is increased within 14 minutes of exposure to 1,25 dihydroxyvitamin $\mathrm{D}_{3}$ (ref. 8).

The intermediary metabolism of vitamin D is also now known to be complex. More than 20 other metabolites have been identified, including $23,25-, 24,25-$ and 25,26-dihydroxyvitamin $D_{3}$, but their role is unknown. The biggest enigma is 24,25 dihydroxyvitamin $\mathrm{D}_{3}$, which under normal physiological conditions is the major circulating dihydroxylated metabolite; it has a long half-life in the circulation, suggesting that it does have some function.

Ideas about the way vitamin $\mathrm{D}$ acts on bone have changed dramatically in the past decade. The principal target cells for 1,25-dihydroxyvitamin $\mathrm{D}_{3}$ in bone are now believed to be the bone-forming osteoblasts rather than the bone-resorbing osteoclasts as previously thought, and the presence of this metabolite seems to be essential for normal bone formation. The stimulatory effect of 1,25dihydroxyvitamin $D_{3}$ on bone resorption may actually be mediated by osteoblastderived factors ${ }^{7}$, but the mechanisms by which the vitamin D system influences bone formation are still unclear.

Even the term vitamin D now seems inappropriate. Normally the diet makes only a small contribution to the body's supply of vitamin D; most is synthesized from 7-dehydrocholesterol in the skin in a physiologically well-regulated process initiated by sunlight ${ }^{10}$.

Despite the remarkable advances that have already been made, there is still much to learn about vitamin $D$. The complexity of its actions has made it difficult to explain satisfactorily the cause of many clinical disorders. It is still not known, for example, why certain groups such as Asian immigrants to the United Kingdom and patients treated with anticonvulsant drugs should be particularly susceptible to rickets or osteomalacia.

Although the fascinating observations by Clements et al. ${ }^{1}$ and by Halloran et al. ${ }^{2}$ help to rationalize these and other clinical observations, they also seem further to expose the limitations of our understanding of the vitamin D system. The observation of Clements et al. that calcium deprivation increases rather than decreases hepatic inactivation of 25 -hydroxyvitamin $D_{3}$, and that this is mediated by 1,25 dihydroxyvitamin $\mathrm{D}_{3}$, is hard to reconcile with the idea that the primary role of the latter is to regulate calcium homoeostasis. A more detailed investigation should help to elucidate the physiological role of the vitamin D system and the function of 1,25 dihydroxyvitamin $D_{3}$ in its many target tissues.

1. Clements, M.R., Johnson, L. \& Fraser, D.R. Nature 325, $62-65(1987)$

2. Halloran, B.P. et al. J. clin. Invest. 78, 622-628 (1986).

3. Bell, N.H., Shaw. S. \& Turner, R.T. J. clin. Invest. 74, 1540-1544 (1984)

4. Blunt, J.W., DeLuca, H.F. \& Schnoes, H.K. Biochemistry $7,3317-3322$ (1968).

Kodicek, E. Lancet i, 325 - 329 (1974)

6. Norman, A.W., Roth, J. \& Orci, L. Endocr. Rev. 3, 331 366 (1982).

7. Shinki, T. et al. J. biol. Chem. 260, 2185-2190 (1985).

8. Nemere, I., Yoshimoto, Y. \& Norman, A.W. Endocrinology 115, 1476 - 1483 (1984)

9. Rodan, G.A. \& Martin, T.J. Calcif-Tissue Int. 33, 349-351 (1981).

10. Fraser, D.R, in Vitamins in Medicine Vol. 1 (eds Barker, B.M. \& Bender, D.A.) 4th edn 42-146 (Heinemann, London, 1980)

Ian Dickson is in the Department of Medicine at the University of Cambridge Clinical School, Addenbrooke's Hospital, Cambridge CB2 $2 Q Q, U K$. 